\title{
Transparency in Measurement Reporting: A Systematic Literature Review of CHI PLAY
}

\author{
LENA FANYA AESCHBACH, SEBASTIAN A. C. PERRIG, LORENA WEDER, KLAUS OPWIS, \\ and FLORIAN BRÜHLMANN, Department for General Psychology and Methodology, University of \\ Basel, Switzerland
}

\begin{abstract}
Measuring theoretical concepts, so-called constructs, is a central challenge of Player Experience research. Building on recent work in $\mathrm{HCI}$ and psychology, we conducted a systematic literature review to study the transparency of measurement reporting. We accessed the ACM Digital Library to analyze all 48 full papers published at CHI PLAY 2020, of those, 24 papers used self-report measurements and were included in the full review. We assessed specifically, whether researchers reported What, How and Why they measured. We found that researchers matched their measures to the construct under study and that administrative details, such as number of points on a Likert-type scale, were frequently reported. However, definitions of the constructs to be measured and justifications for selecting a particular scale were sparse. Lack of transparency in these areas threaten the validity of singular studies, but further compromise the building of theories and accumulation of research knowledge in meta-analytic work. This work is limited to only assessing the current transparency of measurement reporting at CHI PLAY 2020, however we argue this constitutes a fair foundation to assess potential pitfalls. To address these pitfalls, we propose a prescriptive model of a measurement selection process, which aids researchers to systematically define their constructs, specify operationalizations, and justify why these measures were chosen. Future research employing this model should contribute to more transparency in measurement reporting. The research was funded through internal resources. All materials are available on https://osf.io/4xz2v/.
\end{abstract}

CCS Concepts: • Human-centered computing $\rightarrow$ Empirical studies in HCI; HCI theory, concepts and models.

Additional Key Words and Phrases: Transparency, Measurement, Literature review, Methodology, Validity, Survey, Open Science

ACM Reference Format:

Lena Fanya Aeschbach, Sebastian A. C. Perrig, Lorena Weder, Klaus Opwis, and Florian Brühlmann. 2021. Transparency in Measurement Reporting: A Systematic Literature Review of CHI PLAY. Proc. ACM Hum.Comput. Interact. 5, CHI PLAY, Article 233 (October 2021), 21 pages. https://doi.org/10.1145/3474660

\section{INTRODUCTION}

One of the most common ways to collect data in player experience (PX) research is to employ the use of self-report, survey-based instruments. These measurements function by presenting participants with short, declarative statements (e.g., "I enjoyed playing this game very much") or questions followed with a response scale (for example, with options from not at all to very much, never to often, or with a numerical range). The type of data collected through this process is both subjective and

Authors' address: Lena Fanya Aeschbach, lena.aeschbach@unibas.ch; Sebastian A. C. Perrig, sebastian.perrig@unibas.ch Lorena Weder, lorena.weder@unibas.ch; Klaus Opwis, klaus.opwis@unibas.ch; Florian Brühlmann, Department for General Psychology and Methodology, University of Basel, Missionsstrasse 62a, Basel, Switzerland, 4055, florian.bruehlmann@ unibas.ch.

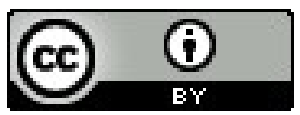

This work is licensed under a Creative Commons Attribution International 4.0 License.

(C) 2021 Copyright held by the owner/author(s).

2573-0142/2021/9-ART233. https://doi.org/10.1145/3474660

Proc. ACM Hum.-Comput. Interact., Vol. 5, No. CHI PLAY, Article 233. Publication date: October 2021. 
quantitative. Subjective meaning, participants are asked to give an honest answer from their own perspective to a question or statement (usually referred to as an item). Quantitative, on the other hand, refers to the form of the data the researcher will receive which is numerical. Because of this, scales and questionnaires offer a multitude of benefits to researchers $[10,13]$. The numerical data can be used for a variety of statistical analyses, while the subjectivity allows to measure complex psychological processes which are not directly observable. For example, motivation or identification, which are both constructs that must be inferred from observed variables. This 'tapping into the subjective' is often necessary to understand the intricate interactions between the players and the games studied. Further, using a valid questionnaire and experimental design, the numerical data collected can be used for statistical analyses that can provide evidence of a cause and effect relationship. When using a reliable questionnaire, the differences between experimental groups on this questionnaire may be attributed to the experiment, rather than errors of measurement In addition, numerical data can be shaped and displayed in a variety of visual representations. This also extends to allow for easier interpretable estimates of magnitude and comparisons of importance.

What is being measured using these measurements are referred to as constructs. A construct has been defined as a theoretical instance of a concept, meaning it is an attribute or idea that is not directly observable or measurable [26]. However, the various benefits associated with measuring constructs using survey scales can only be achieved when we are certain that the questionnaire or scale we employ measures what we have set out to measure. Therefore, when attempting to make a causal statement about an unobservable construct after an experiment, for example motivation, one must be certain that what they measured was actually motivation. This is referred to as the 'validity' of a scale [18].

In regards to this issue, recent research has shown that in psychology there is room for improvement in ensuring the validity of measurements [7]. One first step identified in this regard is the transparency of reporting measurements [22], which must be given to judge whether a measurement is valid within the context it is employed in. Reviewers and readers of a study require specific information about the construct a questionnaire is measuring (e.g. 'Affective State') or how a scale was administered to participants (e.g. 'The Positive and Negative Affect Schedule consists of 10 -item scales ( $1=$ not at all; $5=$ very much) resulting in two sum scores, one for positive affect and one for negative affect.'). Without this information it becomes impossible to evaluate whether the measurement is valid in its given context [3]. This extends to the data and the subsequently drawn conclusions being questionable in their validity. Meaning, an issue with validity in measurement will contaminate all conclusions drawn from the data collected with those measurements [35] It is for this reason researchers, who are invested in the validity of measurements, have called for everyone to engage more strongly with the measurements they use for their studies and the literature surrounding them $[8,47]$.

Within the field of PX research, there are further specific concerns which have been raised. Specifically, certain commonly used questionnaires, such as the Game Experience Questionnaire (GEQ) $[28,42]$, have been questioned in whether or not they are a valid measurement of the construct they propose to measure [11, 29, 31, 38].

It is important to acknowledge that the measurement of subjective experience through selfreported survey scales is only one of several research methods used at CHI PLAY. For example, and this is by no means exhaustive, player behavior and experience is also explored using qualitative methods such as grounded theory [9] and ethnomethodology-informed ethnography [50] or with AI agents in simulations [43]. Indeed, when submitting a paper to the CHI PLAY conference, authors are able to choose from many different contributions beyond research which involves the quantitative measurement of constructs [2]. These contributions are directly derived from the 
many forms of research which has been published in HCI [57]. This highlights that CHI PLAY is a diverse and multidisciplinary community (see also Carter et al.'s Paradigms of Games Research in $\mathrm{HCI}[12])$ and so are the scientific methods used to generate knowledge.

Due to this multidisciplinary field of research, transparency has become an important topic in many different forms. Indeed, transparency has been investigated in regards to sharing data, analytic methods or using preregistration to record possible changes to the research design over time [14] Further, transparency of materials such as source codes [19] and research artifacts have been investigated [55]. However, none of these works has yet reviewed transparency of measurement reporting specifically, as such, further work in regards to transparency of research is needed.

Additionally, best practices on measurement reporting are not commonly shared within the research community. This uncertainty of the measurement quality in PX research is in opposition with the recently expressed aim of the CHI PLAY. Namely the aim that CHI PLAY papers be included in meta-analytic research [36]. If one can not be certain of the validity of a measurement, generalizing the construct measured over multiple studies would be a fruitless endeavor [20]. Therefore, it is crucial that the validity of the measures is investigated and that the measures are reported transparently. This does not only concern self-reported survey scales but also objective measurements. However, reporting and establishing the validity of scales is arguably more challenging than, for instance, of time played or number of levels completed.

The contribution of this systematic literature review is therefore threefold: First, the review of literature provides an accessible discussion of the validity of measurements and its importance and elaborates how this relates to general concerns in regards to the generalizability of studies and consequently their inclusion in meta-analyses. Second, we examine the reporting of survey scales at CHI PLAY 2020. This will provide a first understanding of the state of transparency in the reporting of survey scales within PX research. Third, we provide a model of the measurement selection process for PX researchers to employ. This should aid in achieving transparent measurement reporting, therefore improving the validity and generalizability of findings.

\section{RELATED WORK}

\subsection{Survey scales in research}

Survey scales and other self-reported surveys have often experienced a negative stigma due to the methodological biases which they can potentially introduce [15]. For example, reviewers in organizational psychology often question the legitimacy of self-reported questionnaires in favor of more objective data [13]. However, evidence suggests that frequently discussed biases, such as social desirability, do not have strong, consistent effects [56].

2.1.1 Validity concerns of survey scales. The concerns over self-reported questionnaires can, despite this, still be valid. In specific, user experience (UX) researchers have expressed doubts in regard to whether measurements can accurately capture the experience of users, as empirical data is meaningless without theory [32]. In the broader context of empirical research, substantial method effects threaten the validity of results drawn from measurements. Validity is commonly understood as the appropriateness, meaningfulness and usefulness of the specific inferences made from test scores [5]. DeVellis [18] defines three forms in which these measurements need to prove their validity. First, content validity, which refers to the fact that a scale's content should reflect the conceptual definition applicable to that scale. Second, criterion validity, also sometimes referred to as 'predictive validity', is a theory-neutral form of validity. Criterion validity assesses whether the empirical relationship between a scale or item and a criterion, or 'gold-standard' is strong [18]. Third, construct validity, which places the measurement in relation to other measurements, ensuring that the intended construct is being measured and not an alternative one $[15,40]$. In line 
with previous research, we define constructs as theoretical concepts aimed at organizing and making sense of our environment [40]. These constructs are not directly observable or measurable [26] Construct validity can be evidenced using different statistical methods, however it is impossible to definitively prove construct validity [40]. Despite this, methods exist to provide evidence of construct validity. These methods are generally referred to as the validation of a survey scale. Included in the validation can be an analysis of factorial structure, appropriate reliability measures, mean differences between relevant groups (known-groups validation) or evidencing divergent validity by measuring a construct that is supposed to be distinct [15]. Often this information is present in studies which validate a particular questionnaire. In subsequent studies, which employ the scale, this information is made available to reviewers through references. However, the epistemological discussion surrounding validity, while having impactful consequences for our research, is much deeper than we can discuss in this paper.

Indeed, this paper is instead a first step towards transparency in measurement reporting. We argue that beyond providing evidence of construct validity through reference, researchers have an obligation to provide enough information to judge the specific use of a questionnaire in their study. The content of this information will be dependent on the study. However, information to answer the necessary questions a reviewer might have about the appropriateness of a measurement should be present in all studies which employ questionnaires.

\subsection{General concerns when reporting measurements}

As explained above, the constructs we measure and the measurement we chose for these constructs are a fundamental challenge of every empirical study. In their paper Vornhagen et al. [54] gave a broad idea as to how theoretical variables should be reported in studies. They explain these constructs must be precisely defined, the measurement procedure must be elaborated on and the selection of measurements must be justified. In this section we will review psychological literature on measurements and the reporting of them to explain why this information and more is crucial to ensure transparency of measurement reporting.

\subsection{Defining constructs}

In line with Vornhagen et al. [54] we discuss why precisely defining a construct before its measurement is necessary for interpretable and generalizable findings. Referencing DeVellis [18] conceptualization of content validity, researchers cannot be certain that the content, or items, of a scale is appropriate for their chosen construct if they do not have a clear definition of their construct. Being uncertain about what it is that is being measured, makes it impossible to precisely measure this construct. MacKenzie [35] elaborates how inadequately specifying the conceptual meaning of the study's focal constructs will inherently lead to undermined construct validity. Undermined construct validity further contaminates internal validity and statistical conclusion validity. Meaning that researchers cannot be certain of the results, or the implications drawn from them, which they generate using scales with undermined construct validity. This presents a serious threat to the generalizability of the findings of studies with ill-defined constructs. It further hinders the development of theory, as theory is built upon the building blocks of well-defined constructs [48]

This is an even larger issue, as it has been noted in psychology that certain constructs can be studied for decades and still remain ill-defined [3]. Antonakis [3] details how these ill-defined constructs hinder the advancement of research, as one can never be certain if a study accurately captured the construct they meant to capture. Often, to bridge the troubled waters of ill-defined constructs, researchers will use measurements to theorize about their constructs, rather than using theory to construct their measurements. 
2.3.1 Specifying constructs in theory. Constructs should be specified within a certain theory [24]. If this theory is lacking when measuring constructs, it has further implications. One large issue with relying singularly on statistical processes to assess validity of a measurement is that they can be used while disregarding theory [21] and rendered meaningless because of it [37]. Indeed, this leads to theory being not prospectively described and operationalized. Meaning, theories will lose their predictive value, leading to difficulties when hypothesizing and designing studies.

In their satirical research paper Satchell et al. [45] validate a scale measuring 'Offline Friend Addiction'. They do so by defining the construct of 'Addiction' by the outcomes of the measurement, following the methods of other commonly used social media addiction scales. This leads to a conceptualization of addiction as anything in which people passionately engage in employing repetitive behaviors, whether or not these behaviors are actually pathological. This shows the problematic nature of defining constructs by the scales that measure them. This lack of theory to construct statistically valid questionnaires has been further examined in a study by Maul [37]. Maul finds that meaningless scales using nonsensical terms can be evidenced as valid using methods such as factor analysis, as participants generally answer consistently as long as the same answer format is given. This consistency is not due to actual construct validity, but will satisfy the same statistical criteria as a meaningful survey scale being validated in the same manner. Therefore, statistical processes of validation of survey scales are only meaningful when the scales are built on solid theory supporting them.

2.3.2 Poor Definitions and their Implications. Poor definitions of constructs can be recognized through the following qualities [3]: Constructs should never be defined by their outcomes or ascendents. Further, if a construct is frequently equated to other constructs, it could indicate a lack of divergent validity. Finally extremely broad definitions are also not useful for their precise operationalization. However, often constructs are not defined by the researchers in the first place. What researchers ought to do is to combine the definitions of previous literature to create their own [35]. In contrast to this, many psychological researchers instead provide an overview of previous definitions without defining their own [3]. This makes it impossible for reviewers to judge the accuracy of the selected measurement, as a concept such as 'engagement' could be understood by the authors in multiple different ways and consequently different measurements would be more or less accurate. Ekkekakis and Russell [21] explain that researchers have become desensitized to fundamental issues of imprecise terminology and blurred conceptual distinctions. Due to their frequency, researchers can gloss over such inconsistency and imprecision when reviewing papers. In many disciplines the clear distinction of terms is still a rather young phenomenon. As such it is important to inform researchers who attempt to precisely measure a certain construct of best practice standards when defining constructs [21].

\subsection{Operationalization of constructs}

Constructs are theoretical concepts, which are not directly observable [26]. This means a translation from the theoretical concept into a measurable object has to be created. This translation of something not observable into something observable, something not measurable into something measurable, is referred to as the operationalization of a construct [49]. The idea of operationalism (e.g., [53]) behind the practice of operationalization is an important discussion, but out of scope for this paper. Indeed, we will instead focus on practical concerns when reporting the operationalization of constructs.

How a construct is operationalized can introduce methodological variability. Meaning that difference in measurement presentation or administration can influence the results gathered from 
a survey scale [22]. Indeed, issues such as minor wording changes [34], or the amount of points on a Likert scale [30] can influence the answering behavior from participants in studies.

Further ambiguity around how a measure was precisely operationalized contributes to a lack of continuity in research [22]. This means that researchers who aim to measure precisely the same construct in the same way (e.g., for a close replication), would be unable to and would have to guess, introducing possible differences in results due to methodological variability. Further, should researchers not report exactly how the measurement was operationalized and administered, it is impossible for reviewers and meta-researchers to know how a construct was measured.

\subsection{Measurement selection}

When researchers make the decision to employ a survey scale, they are immediately confronted with yet another decision. Which questionnaire of the many should one use to measure their constructs? When one looks into previous research one can find a multitude of questionnaires measuring common constructs such as enjoyment, so which of those is the most appropriate one? Or the opposite could be true; after looking for a while no questionnaire that fits the construct the researcher has imagined appears. According to Ekkekakis and Russell [21] researchers sometimes like to pretend no such thought process has occurred within them. In those cases it can appear as if a measure appears out of the blue in the measure section with no justification for its presence given Even when justifications for measurements are given they are often superficial. Researchers refer to how commonly a measurement is employed or its age. Neither of these aspects are necessarily indicators of the appropriateness or quality of a questionnaire. Ekkekakis and Russell also describe that researchers continue to use questionnaires which have received valid critiques.

This idea of negligence towards measurement selection is supported by Pedhazur and Pedhazur Schmelkin [40] who note that researchers often treat measurements 'mindless'. Measurements appear to be used simply because they 'exist', with researchers investing naive faith into them.

As discussed before, Ekkekakis and Russel further elaborate how when researchers select a scale, they should understand and agree with the underlying theory of this scale [21]. This is especially important in light of the meaninglessness of statistical validation without a theoretical conceptualization of a construct [37]. This leads to researchers not only using a scale, but also using and supporting the theory which was used to build it, however, the majority of papers do not explicitly and meaningfully discuss this theory they have chosen in tandem with their scale [51].

A further concern lies in a common reason of measurement selection, namely its seniority [21]. Indeed, researchers should not simply 'grandfather in' scales which have been employed before solely on the basis of their previous use. Pedhazur and Pedhazur Schmelkin note that certain scales, such as the F(ascism) scale by Sanford et al. [44] stemming from the 1950s would not be appropriate for use in its original state, as the context in which the items are posed has changed drastically since then [40]. As such, even if scales have been employed previously by other researchers, it is important to note for what reason their use is appropriate in the current study.

Therefore, there is a need for a rigorous selection process for measurements which is reported for each study, so that reviewers can judge the validity of any chosen measurement.

\subsection{Self-developed measures}

So far we have discussed validated questionnaires and their potential issues with validity. However, not for every study conducted in PX has an appropriate measurement already been developed and validated. This is a situation that occurs commonly in $\mathrm{HCI}$ research [6, 41]. In these cases researchers might choose to employ self-developed measures instead. The self-developed measures we refer to function similarly to validated questionnaires [27]. While the use of these self-developed measures is not inherently problematic, often no assessment of the quality criteria, including validity, is made.

Proc. ACM Hum.-Comput. Interact., Vol. 5, No. CHI PLAY, Article 233. Publication date: October 2021. 
As there is no validation study to cite, often no information in regards to construct validity is given. This can make it impossible to judge whether a measurement actually assessed the construct it was intended to. When using self-developed measures, researchers need to take additional care to provide information to make it transparent to reviewers whether their measurement was valid [22]. In contrast, validated questionnaires (should) provide the information to judge their construct validity through citation. Further, a validated questionnaire (should) be worded the same each time it is employed. This greatly increases the ease of comparison between studies and especially the generalizability of findings [27].

Therefore, it is advised to always use validated questionnaires whenever possible. This means that constructs which are measured in the field of PX research using a validated questionnaire should not be measured by self-developed measures in a different study without justification [33].

\section{RESEARCH GOALS}

In line with the previously discussed literature this systematic literature review aims to contribute to the quality of future PX research in the following ways:

We aim to provide a systematic review of the current state of transparency of measurement reporting in PX research. Combining these findings we aim to devise a model of a measurement selection process researchers can employ as a first step towards transparency in measurement reporting in PX.

This study does not aim to identify any questionable measurement practices. We only examine the transparency of measurement reporting necessary to be able to identify potential questionable measurement practices. Further, we aim to not single out specific studies and their authors, but only to provide examples of transparent or intransparent measurement reporting.

\section{METHODS}

We conducted a systematic literature review, which we report on following the PRISMA 2020 checklist [39]. We investigate the current state of transparency of measurement reporting at CHI PLAY 2020. The PRISMA Flowchart detailing all steps of the review can be found on https: //osf.io/xbjw6/.

\subsection{Eligibility and Information source}

For this literature analysis, we collected all research articles, or full papers, published at ACM CHI PLAY $2020(\mathrm{n}=48)$. Excluded from the final sample were all non-full text submissions, this was done to ensure that all papers reviewed are in themselves complete and should include all information in regards to their measurements.

4.1.1 Search strategy and Selection process. We used the ACM Digital Library ${ }^{1}$ to download the full proceedings of CHI PLAY 2020. All 48 papers were coded for their structure and then screened for the inclusion of self-reported measures. When papers included self-reported measures they were further analyzed according to the following code book.

\subsection{Potential Bias}

No risk of bias due to reporting biases (e.g., publication bias) should be present in this study, as our goal is to assess transparent measurement reporting in only published full papers. This is done as to not implicate the research field with intransparency of measurement reporting in papers which did not pass peer-review or are not completed. We acknowledge there is a potential bias as the proceedings of CHI PLAY 2020 could differ in terms of transparency from other years. However,

\footnotetext{
${ }^{1}$ https://dl.acm.org/conference/chi-play/proceedings
} 
the recency of these proceedings should provide us with the current transparency of measurement reporting as a fair basis to assess potential pitfalls.

\subsection{Code book}

Using the questions to promote transparency of measurement reporting proposed in Flake and Fried, we developed an initial code book. The early categories followed six questions proposed by Flake and Fried closely [22]. However, we excluded the investigation into the quantification of the measurements, as the focus of this paper lies in transparency of study design and measurement selection. As such there were codes in the category of 'Construct Definition', 'Construct operationalization', 'Measurement Selection', 'Modification of Measurements' and 'Self-Development of Measurements'. Post-hoc statistical transformations of the scale were not coded for. We developed the code book further in the following way:

- We structured the coding by isolating each construct measured by a paper and investigating the level of transparency of measurement reporting for each one.

- For our purposes, we decided to record a measurement as self-developed when the authors did not cite a source of that measurement.

- We further recorded which scale was cited, in case of a supposed pre-validated questionnaire, to examine whether the cited papers offer evidence of validation.

- As will be elaborated in the results section, many papers did not offer easily accessible information in form of a 'Methods' or 'Measurement' section, as such we included codes which identified the presence of these sections and what other names are given to similar sections.

- We added a text code for the descriptions of modifications made to questionnaires and scales. This was done to record what kind of modifications were reported.

- In line with Flake and Fried we implemented a code to assess whether modifications of questionnaires occurred before or after data collection. However, we found that for some modifications it was implicitly known whether modifications occurred beforehand, we added a secondary code to record these instances.

An example of a code would be:

Construct defined (0/1): Does the construct have a definition in the paper. Quality is not important, any statement of ' $x$ is defined/means/is' should be coded as 1.0 should be coded if there is no definition, no matter how common the construct seems, e.g. 'enjoyment'.

The full code book can be found on https://osf.io/jxbg2/

\subsection{Procedure}

In order to test the quality of the initial code book a randomly selected subset of 5 papers $(10.42 \%)$ was coded by the first author to revise the code book accordingly. We introduced a full-stop into the code book for studies who did not measure a construct using survey scales (50\% or 24 of 48 ). After the code book was finalized, the full sample was coded independently, with the first author coding 30 papers, the second author coding 15 and the third author also coding 15 . This created an overlap of 12 papers coded by two researchers independently with which we calculated inter-rater agreement, this was $86.61 \%$ for all numerical codes. In a secondary step we discussed mismatched codes until consensus was reached and finalized the coding for the interpretation of results. After the initial coding was complete, we wanted to assess whether the given citations for each scale contained evidence of validity, as such the first and third author analyzed these secondary sources in regards to whether they examined the factorial structure of the questionnaire. 


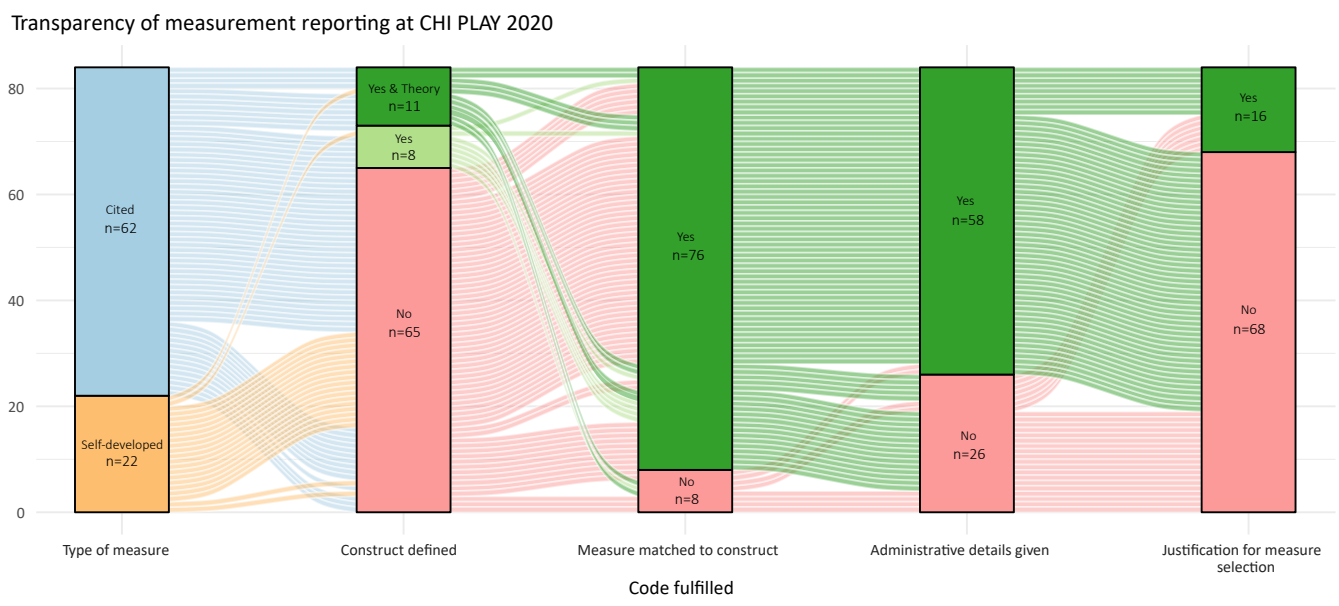

Fig. 1. Number of codes fulfilled for all instances of measurement. $N=84$.

\section{RESULTS}

We present findings in the general order of our code book. We report the results from 24 studies in full, no further eligibility criteria was applied. No data was converted or transformed, as we only counted occurrences of transparency and report on these counts and their percentages. We emphasize again that this review attempts to assess the transparency of measurement reporting in current research, not find questionable measurement practices or lack of validity of measurements. Our full sample was comprised of 48 full papers, of these, $n=24$ or $50.00 \%$ reported measuring at least one construct using some form of self-reported survey instrument.

\subsection{Structure of papers}

To find information about measurements quickly and efficiently, dedicated sections to both the methods and the measurements used aid readers [4]. This can allow researchers to quickly scan papers for this information. We therefore examined whether the paper had sections titled 'methods', 'methodology' or 'method' and further a section titled 'measurements'. Were no such sections present, we instead recorded the presence of a section describing the methods or one describing the measurements and further recorded their names. We found that in the total sample of 48 papers, 23 of 48 papers $(47.92 \%)$ included a section titles method, methods or methodology. Further 22 of 48 papers $(45.83 \%)$ included a section which was similar to methods, but it was named differently, examples of these names include 'Procedure' or 'Experiment N'. Only 3 of 48 (6.25\%) had no section to detail their research methods. We also recorded whether a specific measures subsection was present in the 24 papers in which at least one occurrence of measurement was reported, we found that 12 of 24 papers $(50.00 \%)$ which measured at least one construct, included a measures subsection. On the other hand, 11 of 24 papers $(45.83 \%)$ included a section which included the information about measures, but named differently, with names such as 'Procedure' or 'Tasks'. As discussed above, we recorded these instances as we find a unified language and structure of a research field an important step towards meta-research. 


\subsection{Constructs and Measurements}

In total, we recorded 84 instances of measurement, of those 62 employed cited measurements and 22 employed self-developed measurements (see the first bar 'Type of measure' in Figure 1). We found 67 different constructs. Enjoyment was the most popular construct, with it being measured in six different instances. Most of the constructs were unique, only being measured once throughout the 24 papers $(n=60)$. This breadth was further reflected in the recorded measurements, we found 41 different cited measurements used, while 22 measurements were self-developed by the authors of the papers.

\subsection{Defining constructs}

As described previously, the first step to measure a construct is to define it. Unless a researcher clearly states how a construct is to be understood in the context of their study, it becomes impossible to assess whether their measurement was appropriate. As defining a construct by its measurement is not appropriate either $[3,45]$, researchers need to state a clear definition of how the researched construct is to be understood. Simply matching a construct to a measurement without a proper definition does not provide enough information to readers and reviewers. In total, we found 19 of 84 constructs measured $(22.62 \%)$ were defined. 11 of 84 constructs were specified within a theory (13.1\%). We found that 12 of 24 papers (50\%) defined at least one construct, while only 2 of 24 papers $(8.33 \%)$ defined all of their constructs measured. Examples of definitions of constructs are as follow:

"Self-presence represents the connection between players and their avatars, which guides people to choose what to present via their avatars" (N21).

or for the construct "Creativity":

"Creativity is expressed in different forms depending on the nature of the task and the medium of creative expression figural creativity (e.g., drawing, painting, sculpting) and verbal creativity (writing, storytelling, composition, discourse) [22]. In this work, we focus on figural creativity that is illustrated through a drawing game." (N5).

For cited measurements we found that in 17 of 62 instances $(27.42 \%)$ authors defined the constructs they measured.

We also specifically recorded the percentage of self-developed measures being defined and found 2 of 22 self-developed measures $(9.09 \%)$ were defined. For example, one definition of the construct 'Game Atmosphere' given was:

"The term 'game atmosphere' is used to describe a subtle but important, intangible, generally aesthetic quality in games that leads to emotional immersion." (N36).

or the construct "Purchase Intention", which was defined as:

"[...] the desirability of owning the game [...]" (N1).

5.3.1 Specifying Construct within Theory. For a complete understanding of a construct, the definition needs to be specified within a certain theoretical framework [24]. For this we collected how many authors provided information in regards to which theory their construct is specified in. A construct was considered as derived from theory, if authors gave any reference to the theory in which their definition of a construct is to be understood. We found that 11 of 19 defined constructs (57.9\%), were specified within a theory. One example of being transparent in regards to which theory informs their construct definition was as follows: 
"Based on Goffman's metaphor of theatrical performance[14], the notion of selfpresentation online highlights how identity is portrayed and experienced as a combination of conscious personal choices and specific technological affordances of online social spaces" (N21).

A visualization of the rate of definitions given to measured construct can be found in the second bar ('Construct defined') in Figure 1.

\subsection{Operationalization of constructs}

Two codes were used to record the transparency in regards to the operationalization of constructs. How precisely a construct was operationalized is important to know for the reviewers who need to judge whether a specific measurement is appropriate for the construct. But further, it is vital for potential replications of research, as without it the precise method would be not replicable.

5.4.1 Matching measurements to construct. The first code recorded whether the authors matched their cited measurements to their constructs. We found this to be consistently the case, with 76 of 84 constructs matched to the cited measurement used (90.48\%). An example of a such a matching is:

"Participants' enjoyment was measured using the enjoyment scale items from the Intrinsic Motivation Inventory" (N19).

When self-developed measurements were reported this was done especially consistently with 21 of $22(95.45 \%)$ matching them to the constructs they measured. However when examining cited measurements this was still consistently done in 55 of 62 instances $(88.71 \%)$. This code is represented in the third bar ('Measure matched to construct') in Figure 1.

5.4.2 Administration details. The second code was concerned with whether authors would be transparent about the administration of a measurement. Explanations of administration could include such things as the amount of points on a Likert-type scale or descriptions of the digital environment in which participants were present when filling out the questionnaire. Authors of CHI PLAY also relatively consistently provided this information in their papers. As such, 58 of 84 measurements (69.05\%) included information about administration procedure. A typical description of this administrative procedure was as follows:

"Each subscale includes 4 items, ranked on a four-point Likert scale from "Not at all" to "Completely"” (N18).

Again, this was especially consistent for self-developed measures with administrative details being given in 21 of 22 instances (95.45\%). This was done less consistently for cited measurements with 37 of 62 measurements providing this information (59.68\%). This code is represented in the fourth bar ('Administration details given') in Figure 1.

\subsection{Justification for measurement selection}

As discussed in the related work section, authors should provide a justification for the specific measurement they cited. Justifications could include statements about widespread use or about the validation of a questionnaire. We emphasize that this code was merely about the presence of such a justification and we did not make any judgement about the validity of these claims. We found that authors often neglected to explain why they selected a measurement, with 16 of 84 total measurement choices $(19.05 \%$ ) being justified by researchers. When citing a measurement, researchers justified their selection 16 of 62 the time (25.81\%). Justifications ranged from very simple statements such as:

"a well-validated measure of intrinsic motivation for a task" (N17), 
to more in-depth explanations:

"We opted for this univariate scale because it is less extensive as other scales such as the NASA Task Load Index [25], which might have become laborious given that it needs to be filled out for each task. In addition, previous work [54] indicates that it is reliable and sensitive to small differences in task complexity" (N3).

For self-developed measurements in 0 of 22 instances of measurements $(0.0 \%)$ authors justified why they created their own measurement. This code is represented in the fifth bar ('Justification for measure selection') in Figure 1.

\subsection{Modification of measurements}

We recorded all described instances in which authors made modifications to their measurement. However, it is possible that authors have neglected to describe minor modifications such as changing the phrasing of 'this activity' to 'this game' in questionnaires such as the IMI. We found that 24 of 62 cited measurements $(38.71 \%$ ) were reported as modified. When modification was reported 9 of 24 of modified measurements $(37.50 \%)$ gave a justification for the modification, while 21 of 24 modified measurements $(87.50 \%)$ gave details or examples for the modification. An example of such a description of modification is as follows:

"Furthermore, we adopted the questionnaire to transfer the individual items to our game setting. For example, the original version "I could readily tell when my partner was listening to me." was replaced with "I could readily tell when the game entities were turning their attention to me."" (N12).

Further in 0 of 24 cases $(0.0 \%)$ where modification occurred, authors reported whether this modification was done prior or after data collection, however in 4 of $24(16.67 \%)$ it was implicitly clear whether modification had occurred before or after data collection.

\subsection{Self-developed measurements}

As described above, self-developed measures require more information than validated surveys, as the validation study of a survey provides evidence for construct validity. We found that 12 of 24 of papers $(50.00 \%)$ included self-developed measurements. Of those self-developed measures 17 of 22 (77.27\%) provided details or explanations of their measurement. An example of such a description of a self-developed measure is:

"7-point Likert scale questions regarding the preferred level of realism of the agent

"I would have been more interested in customizing the agent if it was more cartoon-

like/realistic."'(N19).

Authors provided evidence of construct validity for 4.54\% (1 of 22) of the self-developed measures, in this case authors calculated an exploratory factor analysis. The rate of self-developed measures to cited measures is represented in the first bar ('Type of measure') in Figure 1.

\subsection{Validity evidence for cited measurements}

So far we have used the term 'cited measurements' in the results section, rather than 'validated measurements', due it being unknown when only coding the original papers whether the citations provided had any evidence for construct validity as one would assume. In order to assess whether the citations provided evidence of construct validity, we coded the cited papers for all measured constructs. Meaning we searched the 41 cited papers for evidence such as exploratory or confirmatory factor analysis. We found 19 of 41 citations (46.34\%) did not provide any evidence of construct validity. In a tertiary step we attempted to find any papers, not those which were cited by the 
authors of CHI PLAY, which provided construct validity evidence for the employed survey scales. In this manner we could find that 30 of 41 the survey scales $(73.17 \%)$ cited by authors offer evidence of validity at one point or another. However, the scale which was cited and used could differ from the scale which showed evidence of validity. As such, it is unclear to us whether the construct validity reported in those tertiary papers was applicable to the version of the scale employed by the researchers at CHI PLAY 2020.

\section{DISCUSSION}

The first aim of this study is to provide an overview of the current state of transparency of measurement reporting. Our findings show both strengths and weaknesses in regards to transparency. As such we present a discussion of the findings from our systematic literature review of the $\mathrm{CHI}$ PLAY 2020 literature. We note that our data only includes papers published at CHI PLAY 2020, as such these findings do not lend themselves to implicate all of PX research. Instead, we describe current trends found in our data and their broader implications. Further, this research needs to be contextualized in the multidisciplinary field of PX research. This paper does not aim to prescribe the quantitative measurement of constructs as a one-size fits all solution to our field. For example, the contribution of artefact research is important without the empirical data of quantitative measurements. Instead, our aim is to amplify the importance of transparency in measurement reporting and validity of measurements to draw robust conclusions from these empirical findings. With the concerns raised by this paper in mind, researchers might find that their research is not significantly improved by the inclusion of measurements. Therefore, their artefact based research should not include the quantitative measurements of constructs [23,57]. This way, researchers not primarily concerned with the outcomes measured with survey-scales, do not have to implicate their findings with issues of measurement validity.

\subsection{Findings from systematic literature review}

6.1.1 Defining constructs. In our assessment of current transparency of measurement reporting we found that intransparency often begins at the very basic question of what is being measured. Less than a quarter (19 of 84 or $22.62 \%$ ) of all constructs which were measured were defined at all. As shown in the method section, our code did not distinguish between correct, incorrect, complex or simple definitions. A simple statement of what a construct is or means was coded as this construct being defined, yet such statements were absent in most instances of measurement. This means that reviewers will be unable to judge whether a chosen measurement is appropriate for the chosen construct as understood by the authors. As described in the related work, being uncertain or unclear about what is being measured will undermine all results originating from that measurement in their validity $[3,21,35,45]$.

But this lack of definition also extends to the broader context of research. While definitions can not be proven, they need to be consensually considered useful by a research community to make research comparable and cumulative in meta-research [46]. However, such a consensus can only begin to form when researchers consistently invest effort into conceptualizing the constructs they study. PX research is at risk of running into similar issues as researchers in other disciplines in which they attempt to measure complex psychological processes. For example health-behavioral [21] or organizational sciences [3]. Namely a lack of a cohesive understanding of central concepts and how to distinguish them from other similar but not synonymous constructs.

The absence of consistent definitions can further lead to a void of theory due to brittle conceptual building blocks. However, the theory behind constructs is even more rarely reported within our sample of papers. In regards to specifying constructs measured in their respective theory it only occurred in 11 of 84 instances of measurements (13.1\%). This is also problematic in multiple regards. 
In the absence of theory the results of statistical validation procedures such as confirmatory factor analysis become essentially meaningless [21,37]. This, again, undermines construct validity of a measurement and subsequently the results drawn from it. However, these issues are not constrained to the studies in which they occur, but, again, extend into the entire field of PX research and beyond. Especially with the expressed aim to be included in meta-analytic research [36], it is important to build solid theories that can be generalized over all of PX. However, if the constructs are not specified in a specific theory conceptually, studying them and reporting on their effects will not aid in theory-building [24].

6.1.2 Operationalization of constructs. We found that it was often transparent how a construct was measured. Meaning, the operationalization of constructs was often easy to discern both for cited and self-developed measurements. When using a measurements, researchers would most often ( 76 of 84 or $90.48 \%$ ) match it to the construct they measured. This way reviewers can discern whether they find the selected measurement appropriate for the construct given. Further, details relating to administration such as the points of the Likert-type scale employed were often ( 58 of 84 or $69.05 \%$ ) given. This shows that researchers at CHI PLAY are aware of the requirements of providing such information. This also allows for reviewers to assess whether the measurements used were implemented appropriately.

When researchers developed their own measurements, they gave information of the actual content of their scale. Meaning they provided examples of the items they used or gave explanations in regards to them, making it possible to understand how they measured their proposed construct (17 of 22 or $77.27 \%$ ).

This is reflected in the coding of the modifications done to cited measurements that were also commonly described by the researchers. This is important, as statistical processes that evidence construct validity can be made non-applicable by severe modifications [22]. Describing the kind of modification that was done for a measurement allows for reviewers to judge the appropriateness and whether the original validation still applies or researchers need to validate their own modified version.

6.1.3 Justification for measurement selection. A second issue is the intransparency in regards to the process of measurement selection, or why the chosen construct was measured in this way. A fifth (16 of 84 or $19.05 \%$ ) of all measurements stated any kind of reason why they selected their specific measurement. For example, despite recent research questioning its validity [11, 29, 31, 38], the GEQ $[28,42]$ was used in our sample with no justification as to why (N28). This can indicate a lack of a rigorous measurement selection process in which authors make informed decisions as to which measurement is best suited for their chosen construct.

6.1.4 Modification of measurements. In regards to modifications, we also found that no researcher explicitly stated whether a modification occurred before or after data collection. In case of changed wordings, this can be considered negligible, as implicitly it is known that modification had to occur before data collection to impact the findings, but for other modifications it is imperative to know in what part of the research process they happened. Dropping individual items or dimensions of scales during analysis could result in accidental p-hacking [25]. Similarly, less than half of the time ( 9 of 24 or $37.50 \%$ ) researchers reported why they had modified the measurements they used. Any modifications and justifications as to why they had to occur would also be part of a transparently reported measurement selection process. However, if modifications occur before data collection, are justified and pose no threat to construct validity, they can be beneficial to the study.

6.1.5 Self-developed measures. In comparison to similar research in UX studies, we found a similar amount of studies employed self-developed measures. In their study Pettersson et al. find that $53 \%$ 
of papers used at least one self-developed measure, while in our sample we found a rate of $50 \%$ [41]. When examining the use of self-developed measurements, further issues arrived. We found that some researchers developed their own measurements for constructs that other researchers at CHI PLAY 2020 employed a validated questionnaire for (e.g., 'Enjoyment'), while other times a self-developed questionnaire was used for constructs for which validated survey scales exist (e.g., 'Satisfaction'). As no researcher provided reasoning for why they developed their own measurement, reviewers and meta-research can only guess why they chose to create their own measurement. The reasonings could be issues of implementation (e.g. length of a validated survey scale) or theoretical (e.g. different understandings of how a construct should be defined), but it remains unclear to readers of the paper. Justification for why a self-developed measurement was employed should always be given [22], but are especially important when validated measurements already exist for the same construct [27]. Further, in only one instance (1 of 22 or $4.54 \%$ ) did authors provide an investigation into the factorial structure of a self-developed measure. Meaning for only one self-developed measurement evidence of construct validity was reported. This directly impacts how certain we can be of the implications drawn from the results of the measurement, as reviewers and meta-researchers can not evaluate whether the measurement measured what it set out to measure [18].

\subsection{Further implications of intransparency}

As this study investigates transparency, a lack thereof leads to a number of possible implications depending on the information which was not reported by researchers. We aim to report on the most important possible implications of potential issues in this section.

6.2.1 Jingle-Jangle Fallacy. Intransparency in regards to both definitions and selection can lead to the so-called 'jingle-jangle fallacies' [22]. The jingle fallacy refers to the issue in which researchers assume measurements with similar names measure the same construct, while that is not actually true. The jangle fallacy refers to the inverse, two measures measuring supposedly different constructs because their names are different, while in reality they measure the same construct. We find that there is a possibility for jingle-jangle fallacies to occur as certain constructs, such as 'Enjoyment', are measured with different scales without justification or definition. With a lack of explicit definitions and justification of measurement selection, it is difficult for reviewers to know if researchers who measured enjoyment with the Intrinsic Motivation Inventory (IMI) [17] conceptualize and theorize it in the same way as those who measure it with the Player Experience Inventory (PXI) [1]. As such, researchers also need to start defining their constructs and justifying their selection of measurement to address potential jingle-jangle fallacies.

6.2.2 Confusing and Incorrect Citations. Another confusing issue arrived when recording the citations of questionnaires. We found certain authors cited studies that offered no evidence of construct validity for the questionnaire they used (46.34\% or 19 of 41). Alternatively, some citations were simply incorrect, such as in the case of the GEQ, which has a history of confusing citations [31]. We also found researchers citing different studies for measurements of the same name. This can lead to confusion as it is unclear if there are multiple versions of the scale validated under the same name. All of these issues regarding citation made it near impossible to assess in certain cases whether a survey scale that was employed had been validated previously or whether evidence of construct validity for this survey scale was simply lacking. This issue will be similar for reviewers who try to judge the validity of a scale. A reviewer could assume that a citation of a scale means that this scale has been validated or else the researcher would not employ it without providing their own validation procedures. Therefore, we urge researchers at CHI PLAY to be careful with 
citations and to not report a survey scale which lacks evidence for construct validity in the same manner as one which does offer such evidence of validity.

\subsection{Measurement Selection Model}

The second research goal was to formulate a prescriptive model of the measurement selection process (see Figure 2) in line with research models devised by Guest and Martin [24] or Ekkekakis and Russell [21]. We believe that researchers employing our model will promote transparency in regards to aspects of measurements which are currently seldom reported systematically on in CHI PLAY literature, such as definitions of constructs and justifications for measurements. Improved transparency of measurement reporting in turn will aid reviewers and meta-researchers to fully grasp the entire design of a study to judge its appropriateness or inclusion in any given meta-analysis.

6.3.1 Defining constructs: What are you measuring? In line with the discussed literature in this study $[3,35]$, we understand measurement as beginning with the conceptual and theoretical definition of a construct, rather than the operationalization of that construct. While in our literature research we did not investigate the quality of definitions given, there are still certain aspects that make something a good definition.

The choice of construct should be given a lot of weight. Researchers need to be certain that this construct was the most appropriate for the given context. As such researchers should also remain specific and not use near synonymous terms within the same study [21]. Similar terms such as 'Immersion' or 'Engagement' should therefore not be used interchangeably in the same study.

Once a specific construct is decided on, the precise definition should be reported in the study. Good definitions should fulfill a number of standards. Firstly, good definitions should specify the constructs conceptual theme and be unambiguous. Meaning they need to be clearly distinguished from related constructs. Further, if the construct is multidimensional, the relationship between the dimensions need to be explained [35]. It is also important for researchers to take ownership over the definition they use in their study. Researchers should give a statement how they defined the construct in their study, not simply how it has been defined previously [3].

This definition further needs to be specified within a theory and not simply stand for itself [24]. Understanding which theoretical underpinnings inform a construct is necessary to fully define a construct.

6.3.2 Operationalization of constructs: How are you measuring? Once a construct is defined both conceptually and theoretically, researchers can move on to considerations how exactly they will measure this construct. Should a validated questionnaire exist with the same conceptual and theoretical background as the construct defined, this should always be chosen over developing your own survey scale [27]. As changing numbers of Likert scales can change the results collected from experiments, researchers should use the validated format [16]. Further modifications, including the wording of questions, translation or time specification in recall periods, can also impact their validity [30]. As such they must be carefully considered and fully reported.

Should no appropriate validated questionnaire exist, researchers can develop their own measurement. These measurements must also be in line with the theoretical considerations of the construct.

6.3.3 Justification of measurement selection: Why are you measuring this way? All of the previous considerations combined should give the first part of the reasoning as to why a certain measure was selected or developed. In addition to considerations in regards to definition, theory, administration and operationalization as described above, a comprehensive report on validity must be given. This 


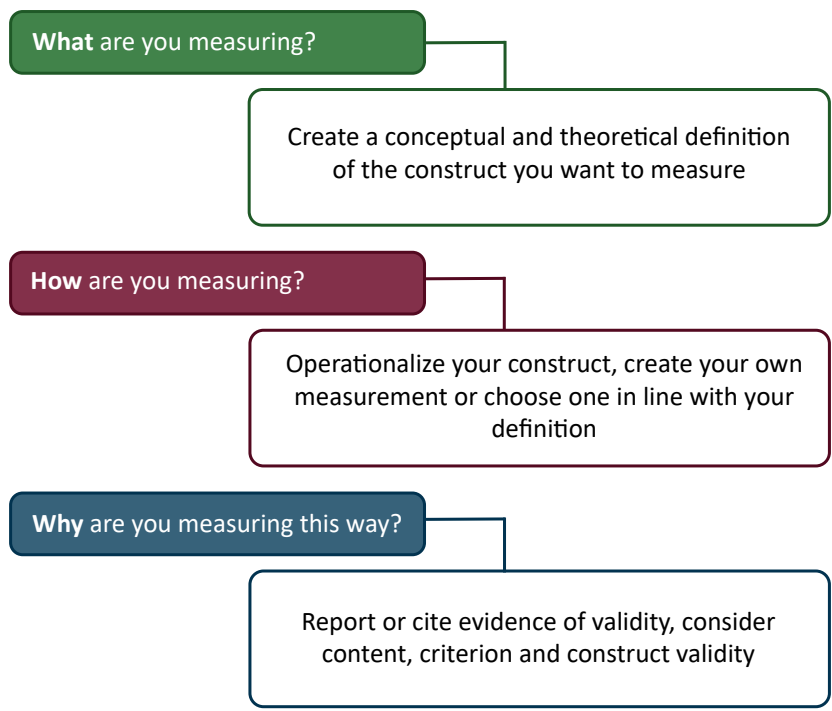

Fig. 2. Model of a measurement selection process

includes considerations of content, criterion and construct validity [18]. When using a validated questionnaire, these can be found in validation studies of those questionnaires. However, selfdeveloped questionnaires must be validated according to best practice guidelines within the study in which its results are reported and interpreted.

6.3.4 Summary. The answers to all questions posed in our model of the measurement selection process should be reported in each study measuring at least one construct using a survey scale. Ideally, this should result in the transparent reporting of information in regards to definition, theory, administration, operationalization, validation and justification of a measurement used.

As a result of reporting all of these considerations, researchers can achieve transparent measurement reporting. Employing a prescriptive model of measurement selection should improve the current state of transparency, especially for presently neglected aspects such as definitions of constructs and justifications for selected measurements.

\subsection{Limitations \& Future Research}

We note a few limitations associated with this study. Firstly, for our systematic literature review we solely analyzed the literature published in one year of the ACM CHI PLAY conference. Namely, the latest one of CHI PLAY 2020. However, we believe for our purposes of providing a current state of transparency in measurement reporting, it surmises an appropriate sample. Further, we chose CHI PLAY 2020 as we expect research to improve over time and we expect the most recent year to be the most transparent year in measurement reporting. This should provide a fair basis for issues in regards to the current state of transparency in measurement reporting

As with all literature reviews, the possibility exists for coder error or individual differences in how specific instances are understood and coded, even with especially diligent coding. As this study does not attempt to single out individual papers or make examples of them, but rather show an overall trend, any error which might have occurred should be within margin. However, in order to mitigate these concerns we assessed inter-rater reliability. 
Further, this review was not preregistered, however the code book followed Flake and Fried's methodology closely and all changes to this have been detailed in this paper under the code book section. The code book and coding are further in full available on https://osf.io/4xz2v/.

For our purposes we did not differentiate between atheoretical and theoretical measures as defined by previous research [18]. Although it can be criticized that we judged measures which are supposedly atheoretical on whether they are rooted in theory, we found no paper explicitly state that certain measures are atheoretical. As such it would be our responsibility of judging the intent of authors. This was something we expressly attempted to avoid with our focus on transparency and therefore this consideration is out of scope for this study.

In regards to future research we believe the continued publication of meta-research such as systematic literature reviews, especially with a focus on popular methodologies will aid in both the quality and the ensured progress of the research field.

We further believe it is imperative for PX research to build discussions surrounding the conceptual and theoretical underpinnings of central constructs, with the eventual end goal of a conceptual consensus. Previous research has already noted the need for PX research to move beyond the constructs and methods we can 'borrow' from psychology and other UX to give us legitimacy [52]. Instead, we need to establish new language to discuss games more holistically. For this, researchers need to carefully consider which constructs, theories and measurements they borrow from psychology and other related fields. Further, we find it necessary for PX to grow its own vocabulary and theoretical underpinnings in addition borrowing conceptual and theoretical understandings from established and previously used survey-scales. To achieve this goal, explicit discussions of constructs and theory are a necessary future step.

Lastly, investigations into the validity of questionnaires as well as the creation of new, well validated questionnaires on the basis of theory, will continue to be important to ensure the high quality of PX research published at CHI PLAY.

\section{CONCLUSION}

The measurement of theoretical concepts, or constructs, is an integral part of PX research. The use of survey scales is a common way for researchers to gain data about these constructs through measuring them. Transparency in regards to how these measurements were carried out is vital to judge the validity of a study. However, in our methodological review of 24 full papers we found that in many instances of measurements, information in regards to definition of a construct and justification as to why a certain measurement was selected, was lacking. In comparison, details in regards to operationalization of a construct were commonly included in a paper. To address these issues we present findings as to how they can threaten the validity of a study and introduce a prescriptive model of the measurement selection process. Following the model should encourage and aid researchers in treating the measurement of constructs with the weight it deserves and report on their methods transparently. This will not only improve the quality of single studies, but rather aid in generalizing findings and future meta-analytical work in PX research.

\section{DATA AVAILABILITY}

The data for this systematic review is openly available under https://osf.io/4xz2v/. Including the full coding of all constructs, the coding of the cited measures and their validation, the citations for all coded papers, the citations for the cited measurements, the code book, a list of all the constructs found and R scripts used for analysis. 


\section{AUTHOR CONTRIBUTION}

LFA, FB and SACP conceived the initial idea. LFA designed the literature review and the initial code book. SACP and FB gave feedback to the code book's iterations. LFA tested the initial and iterated code books and created iterations until the final code book was finished. LFA, SACP and LW coded the CHI PLAY 2020 papers. LFA wrote the draft. LFA, SACP, LW, KO and FB contributed to the final version.

\section{ACKNOWLEDGEMENTS}

We thank the reviewers for their helpful feedback. Special thanks to Laetitia Woelfle and Nestor for their support during the writing of this paper. This work was funded internally.

\section{REFERENCES}

[1] Vero Vanden Abeele, Katta Spiel, Lennart Nacke, Daniel Johnson, and Kathrin Gerling. 2020. Development and validation of the player experience inventory: A scale to measure player experiences at the level of functional and psychosocial consequences. International Journal of Human-Computer Studies 135 (2020), 102-370. https: //doi.org/10.1016/j.ijhcs.2019.102370

[2] CHI PLAY ACM. 2021. Contribution Types and Evaluation Criteria. https://chiplay.acm.org/2021/table-1/

[3] John Antonakis, Nicolas Bastardoz, Philippe Jacquart, and Boas Shamir. 2016. Charisma: An Ill-Defined and IllMeasured Gift. Annual Review of Organizational Psychology and Organizational Behavior 3, 1 (Mar. 2016), 293-319. https://doi.org/10.1146/annurev-orgpsych-041015-062305

[4] Mark Appelbaum, Harris Cooper, Rex B. Kline, Evan Mayo-Wilson, Arthur M. Nezu, and Stephen M. Rao. 2018. Journal article reporting standards for quantitative research in psychology: The APA Publications and Communications Board task force report. American Psychologist 73, 1 (2018), 3-25. https://doi.org/10.1037/amp0000191

[5] American Educational Research Association, American Psychological Association, National Council on Measurement in Education, Joint Committee on Standards for Educational, and Psychological Testing (US). 1999. Standards for educational and psychological testing. Amer Educational Research Assn, Washington, DC, USA.

[6] Javier A. Bargas-Avila and Kasper Hornbæk. 2011. Old Wine in New Bottles or Novel Challenges: A Critical Analysis of Empirical Studies of User Experience. In Proceedings of the SIGCHI Conference on Human Factors in Computing Systems (Vancouver, BC, Canada) (CHI '11). Association for Computing Machinery, New York, NY, USA, 2689-2698. https://doi.org/10.1145/1978942.1979336

[7] Adam E. Barry, Beth Chaney, Anna K. Piazza-Gardner, and Enmanuel A. Chavarria. 2014. Validity and Reliability Reporting Practices in the Field of Health Education and Behavior: A Review of Seven Journals. Health Education \& Behavior 41, 1 (2014), 12-18. https://doi.org/10.1177/1090198113483139

[8] Denny Borsboom. 2006. The attack of the psychometricians. Psychometrika 71, 3 (Sep. 2006), 425-440. https: //doi.org/10.1007/s11336-006-1447-6

[9] Katreen Boustani, Anne C. Tally, Yu Ra Kim, and Christena Nippert-Eng. 2020. Gaming the Name: Player Strategies for Adapting to Name Constraints in Online Videogames. In Proceedings of the Annual Symposium on Computer-Human Interaction in Play (Virtual Event, Canada) (CHI PLAY '20). Association for Computing Machinery, New York, NY, USA, 120-131. https://doi.org/10.1145/3410404.3414259

[10] Florian Brühlmann and Elisa D. Mekler. 2018. Surveys in Games User Research. In Games User Research, Anders Drachen, Pejman Mirza-Babaei, and Lennart Nacke (Eds.). Oxford University Press, Oxford, Chapter 9, 141-162.

[11] Florian Brühlmann and Gian-Marco Schmid. 2015. How to Measure the Game Experience?: Analysis of the Factor Structure of Two Questionnaires. In Proceedings of the 33rd Annual ACM Conference Extended Abstracts on Human Factors in Computing Systems. ACM, New York, NY, USA, 1181-1186. https://doi.org/10.1145/2702613.2732831

[12] Marcus Carter, John Downs, Bjorn Nansen, Mitchell Harrop, and Martin Gibbs. 2014. Paradigms of Games Research in HCI: A Review of 10 Years of Research at CHI. In Proceedings of the First ACM SIGCHI Annual Symposium on Computer-Human Interaction in Play (Toronto, Ontario, Canada) (CHI PLAY '14). Association for Computing Machinery, New York, NY, USA, 27-36. https://doi.org/10.1145/2658537.2658708

[13] David Chan. 2009. So why ask me? Are self-report data really that bad. In Statistical and methodological myths and urban legends: Doctrine, verity and fable in the organizational and social sciences, Charles E Lance and Robert J Vandenberg (Eds.). Taylor \& Francis, Abingdon, UK, Chapter 13, 309-336.

[14] Lewis L. Chuang and Ulrike Pfeil. 2018. Transparency and Openness Promotion Guidelines for HCI. Association for Computing Machinery, New York, NY, USA, 1-4. https://doi.org/10.1145/3170427.3185377 
[15] James M. Conway and Charles E. Lance. 2010. What Reviewers Should Expect from Authors Regarding Common Method Bias in Organizational Research. Journal of Business and Psychology 25, 3 (May 2010), 325-334. https: //doi.org/10.1007/s10869-010-9181-6

[16] John Dawes. 2008. Do Data Characteristics Change According to the Number of Scale Points Used? An Experiment Using 5-Point, 7-Point and 10-Point Scales. International fournal of Market Research 50, 1 (Jan. 2008), 61-104. https: //doi.org/10.1177/147078530805000106

[17] Edward L. Deci and Richard M. Ryan. 2003. Intrinsic motivation inventory. Self-determination theory 267 (2003).

[18] Robert F. DeVellis. 2016. Scale development: Theory and applications. Vol. 26. Sage publications, Los Angeles, CA, USA.

[19] Florian Echtler and Maximilian Häußler. 2018. Open Source, Open Science, and the Replication Crisis in HCI. Association for Computing Machinery, New York, NY, USA, 1-8. https://doi.org/10.1145/3170427.3188395

[20] Matthias Egger, George Davey Smith, and Jonathan AC Sterne. 2001. Uses and abuses of meta-analysis. Clinical Medicine 1, 6 (2001), 478. https://doi.org/10.7861/clinmedicine.1-6-478

[21] Panteleimon Ekkekakis and James A. Russell. 2013. The Measurement of Affect, Mood, and Emotion: A Guide for Health-Behavioral Research. Cambridge University Press, Cambridge, UK. https://doi.org/10.1017/CBO9780511820724

[22] Jessica Kay Flake and Eiko I. Fried. 2020. Measurement Schmeasurement: Questionable Measurement Practices and How to Avoid Them. Advances in Methods and Practices in Psychological Science 3, 4 (2020), 456-465. https: //doi.org/10.1177/2515245920952393

[23] Saul Greenberg and Bill Buxton. 2008. Usability Evaluation Considered Harmful (Some of the Time). In Proceedings of the SIGCHI Conference on Human Factors in Computing Systems (Florence, Italy) (CHI '08). Association for Computing Machinery, New York, NY, USA, 111-120. https://doi.org/10.1145/1357054.1357074

[24] Olivia Guest and Andrea E. Martin. 2021. How Computational Modeling Can Force Theory Building in Psychological Science. Perspectives on Psychological Science 16, 4 (2021), 789-802. https://doi.org/10.1177/1745691620970585 arXiv:https://doi.org/10.1177/1745691620970585

[25] Megan L. Head, Luke Holman, Rob Lanfear, Andrew T. Kahn, and Michael D. Jennions. 2015. The Extent and Consequences of P-Hacking in Science. PLOS Biology 13, 3 (Mar. 2015), 1-15. https://doi.org/10.1371/journal.pbio. 1002106

[26] Kenneth D Hopkins. 1998. Educational and psychological measurement and evaluation. Pearson, London, UK.

[27] Kasper Hornbæk. 2006. Current practice in measuring usability: Challenges to usability studies and research. International fournal of Human-Computer Studies 64, 2 (2006), 79-102. https://doi.org/10.1016/j.ijhcs.2005.06.002

[28] Wijnand IJsselsteijn, Yvonne De Kort, and Karolien Poels. 2013. The Game Experience Questionnaire. Eindhoven: Technische Universiteit Eindhoven.

[29] Daniel Johnson, M. John Gardner, and Ryan Perry. 2018. Validation of two game experience scales: The Player Experience of Need Satisfaction (PENS) and Game Experience Questionnaire (GEQ). International fournal of HumanComputer Studies 118 (2018), 38 - 46. https://doi.org/10.1016/j.ijhcs.2018.05.003

[30] E. F. Juniper. 2009. Validated questionnaires should not be modified. European Respiratory fournal 34, 5 (Oct. 2009), 1015-1017. https://doi.org/10.1183/09031936.00110209

[31] Effie L.-C. Law, Florian Brühlmann, and Elisa D. Mekler. 2018. Systematic Review and Validation of the Game Experience Questionnaire (GEQ) - Implications for Citation and Reporting Practice. In Proceedings of the 2018 Annual Symposium on Computer-Human Interaction in Play (Melbourne, VIC, Australia) (CHI PLAY '18). Association for Computing Machinery, New York, NY, USA, 257-270. https://doi.org/10.1145/3242671.3242683

[32] Effie L.-C. Law, Paul van Schaik, and Virpi Roto. 2014. Attitudes towards user experience (UX) measurement. International fournal of Human-Computer Studies 72, 6 (2014), 526 - 541. https://doi.org/10.1016/j.ijhcs.2013.09.006 Interplay between User Experience Evaluation and System Development.

[33] James R. Lewis. 2014. Usability: Lessons Learned ... and Yet to Be Learned. International fournal of Human-Computer Interaction 30, 9 (2014), 663-684. https://doi.org/10.1080/10447318.2014.930311

[34] Elizabeth F. Loftus and Guido Zanni. 1975. Eyewitness testimony: The influence of the wording of a question. Bulletin of the Psychonomic Society 5, 1 (Jan. 1975), 86-88. https://doi.org/10.3758/bf03336715

[35] Scott B. MacKenzie. 2003. The Dangers of Poor Construct Conceptualization. fournal of the Academy of Marketing Science 31, 3 (Jun. 2003), 323-326. https://doi.org/10.1177/0092070303031003011

[36] Regan Mandryk. 2020. PACM Statement. https://chiplay.acm.org/2021/pacm/

[37] Andrew Maul. 2017. Rethinking Traditional Methods of Survey Validation. Measurement: Interdisciplinary Research and Perspectives 15, 2 (2017), 51-69. https://doi.org/10.1080/15366367.2017.1348108

[38] Kent L. Norman. 2013. GEQ (Game Engagement/Experience Questionnaire): A Review of Two Papers. Interacting with Computers 25, 4 (Mar. 2013), 278-283. https://doi.org/10.1093/iwc/iwt009

[39] Matthew J. Page, Joanne E. McKenzie, Patrick M. Bossuyt, Isabelle Boutron, Tammy C. Hoffmann, Cynthia D. Mulrow, Larissa Shamseer, Jennifer M. Tetzlaff, Elie A. Akl, Sue E. Brennan, Roger Chou, Julie Glanville, Jeremy M. Grimshaw, Asbjørn Hróbjartsson, Manoj M. Lalu, Tianjing Li, Elizabeth W. Loder, Evan Mayo-Wilson, Steve McDonald, Luke A. 
McGuinness, Lesley A. Stewart, James Thomas, Andrea C. Tricco, Vivian A. Welch, Penny Whiting, and David Moher. 2021. The PRISMA 2020 statement: an updated guideline for reporting systematic reviews. Systematic Reviews 10, 1 (29 Mar 2021), 89. https://doi.org/10.1186/s13643-021-01626-4

[40] Elazar J Pedhazur and Liora Pedhazur Schmelkin. 2013. Measurement, design, and analysis: An integrated approach psychology press, Hove, East Sussex, UK.

[41] Ingrid Pettersson, Florian Lachner, Anna-Katharina Frison, Andreas Riener, and Andreas Butz. 2018. A Bermuda Triangle? A Review of Method Application and Triangulation in User Experience Evaluation. Association for Computing Machinery, New York, NY, USA, 1-16. https://doi.org/10.1145/3173574.3174035

[42] Karolien Poels, Yvonne De Kort, and Wijnand IJsselsteijn. 2007. D3.3 : Game Experience Questionnaire: development of a self-report measure to assess the psychological impact of digital games. Eindhoven: Technische Universiteit Eindhoven.

[43] Shaghayegh Roohi, Asko Relas, Jari Takatalo, Henri Heiskanen, and Perttu Hämäläinen. 2020. Predicting Game Difficulty and Churn Without Players. In Proceedings of the Annual Symposium on Computer-Human Interaction in Play (Virtual Event, Canada) (CHI PLAY '20). Association for Computing Machinery, New York, NY, USA, 585-593. https://doi.org/10.1145/3410404.3414235

[44] R Nevitt Sanford, Theodor W Adorno, Else Frenkel-Brunswik, and Daniel J Levinson. 1950. The measurement of implicit antidemocratic trends. The authoritarian personality (1950), 222-279.

[45] Liam P. Satchell, Dean Fido, Craig A. Harper, Heather Shaw, Brittany Davidson, David A. Ellis, Claire M. Hart, Rahul Jalil, Alice Jones Bartoli, Linda K. Kaye, Gary L. J. Lancaster, and Melissa Pavetich. 2020. Development of an OfflineFriend Addiction Questionnaire (O-FAQ): Are most people really social addicts? Behavior Research Methods 52, 5 (24 Sep 2020). https://doi.org/10.3758/s13428-020-01462-9

[46] Klaus R. Scherer. 2005. What are emotions? And how can they be measured? Social Science Information 44, 4 (2005), 695-729. https://doi.org/10.1177/0539018405058216

[47] Donald Sharpe. 2013. Why the resistance to statistical innovations? Bridging the communication gap. Psychological Methods 18, 4 (2013), 572-582. https://doi.org/10.1037/a0034177

[48] Pamela Shoemaker, James W. Tankard, and Dominic L. Lasorsa. 2004. Theoretical concepts: The building blocks of theory. In How to Build Social Science Theories. SAGE Publications, Inc., Los Angeles, CA, 15-36. https://doi.org/10. 4135/9781412990110

[49] Brent D. Slife, Casey D. Wright, and Stephen C. Yanchar. 2016. Using Operational Definitions in Research: A BestPractices Approach. The fournal of Mind and Behavior 37, 2 (2016), 119-139. http://www.jstor.org/stable/44631540

[50] Velvet Spors, Gisela Reyes Cruz, H. R. Cameron, Martin Flintham, Pat Brundell, and David Murphy. 2020. Plastic Buttons, Complex People: An Ethnomethodology-Informed Ethnography of a Video Game Museum. In Proceedings of the Annual Symposium on Computer-Human Interaction in Play (Virtual Event, Canada) (CHI PLAY '20). Association for Computing Machinery, New York, NY, USA, 594-605. https://doi.org/10.1145/3410404.3414234

[51] April Tyack and Elisa D. Mekler. 2020. Self-Determination Theory in HCI Games Research: Current Uses and Open Questions. In Proceedings of the 2020 CHI Conference on Human Factors in Computing Systems (Honolulu, HI, USA) (CHI '20). Association for Computing Machinery, New York, NY, USA, 1-22. https://doi.org/10.1145/3313831.3376723

[52] April Tyack and Elisa D. Mekler. 2021. Off-Peak: An Examination of Ordinary Player Experience. In Proceedings of the 2021 CHI Conference on Human Factors in Computing Systems (Yokohama, Japan) (CHI '21). Association for Computing Machinery, New York, NY, USA, Article 115, 12 pages. https://doi.org/10.1145/3411764.3445230

[53] Elina Vessonen. 2020. Respectful operationalism. Theory \& Psychology 31, 1 (2020), 84-105. https://doi.org/10.1177/ 0959354320945036

[54] Jan B. Vornhagen, April Tyack, and Elisa D. Mekler. 2020. Statistical Significance Testing at CHI PLAY: Challenges and Opportunities for More Transparency. In Proceedings of the Annual Symposium on Computer-Human Interaction in Play. ACM, New York, NY, USA, 4-18. https://doi.org/10.1145/3410404.3414229

[55] Chat Wacharamanotham, Lukas Eisenring, Steve Haroz, and Florian Echtler. 2020. Transparency of CHI Research Artifacts: Results of a Self-Reported Survey. In Proceedings of the 2020 CHI Conference on Human Factors in Computing Systems (Honolulu, HI, USA) (CHI '20). Association for Computing Machinery, New York, NY, USA, 1-14. https: //doi.org/10.1145/3313831.3376448

[56] Larry J. Williams and Stella E. Anderson. 1994. An Alternative Approach to Method Effects by Using Latent-Variable Models: Applications in Organizational Behavior Research. Journal of Applied Psychology 79, 3 (1994), 323 - 331. https://doi.org/10.1037/0021-9010.79.3.323

[57] Jacob O. Wobbrock and Julie A. Kientz. 2016. Research Contributions in Human-Computer Interaction. Interactions 23, 3 (Apr. 2016), 38-44. https://doi.org/10.1145/2907069

Received February 2021; revised June 2021; accepted July 2021 